\title{
Viscosity of Magnetite-Toluene Nanofluids: Dependence on Temperature and Nanoparticle Concentration
}

\author{
Rahul Singh, ${ }^{1}$ Oswaldo Sanchez, ${ }^{2}$ Suvojit Ghosh, ${ }^{3}$ Naveen Kadimcherla, ${ }^{1}$ Swarnendu Sen ${ }^{4}$ and \\ Ganesh Balasubramanian ${ }^{1, \Psi}$ \\ ${ }^{1}$ Department of Mechanical Engineering, Iowa State University, Ames, IA, USA \\ ${ }^{2}$ Department of Mathematical Sciences, Morningside College, Sioux City, IA, USA \\ ${ }^{3}$ Department of Engineering Physics, McMaster University, Hamilton, ON, Canada \\ ${ }^{4}$ Department of Mechanical Engineering, Jadavpur University, Kolkata, West Bengal, India
}

${ }^{\Psi}$ Corresponding author

Address: 2092 Black Engineering Building, Ames, IA 50011, USA.

Phone: 515-294-9226, Email: bganesh@iastate.edu 


\begin{abstract}
We examine the dependence of the viscosity of nanofluids, comprised of magnetite nanoparticles dispersed in toluene, on particle concentration and temperature. The nanofluid viscosity increases monotonically with particle concentration. We show that although the nanoparticles aggregate to form clusters with increasing concentration, the cluster size is fairly monodisperse and hence the viscosity can be expressed as a function of only the particle concentration. The viscosity of the nanofluid is found to decrease with temperature, similar to the characteristics of the carrier liquid. We describe these dependencies through an empirical correlation, since the observations are useful to employ such nanofluids in engineering applications.
\end{abstract}

Keywords: Nanofluid, Viscosity, Temperature dependence, Particle volume fraction 


\section{Introduction}

A typical nanofluid comprises colloidal nanoparticles (NPs) of a solid material dispersed in a liquid. Crystalline metal or metal oxide NPs are often used as the dispersed phase to engineer nanofluids for thermal management applications [1]. This is because (1) such NPs have an inherently high thermal conductivity and (2) the small size of the NPs (diameter $d \sim 10^{0}-10^{2}$ $\mathrm{nm}$ ) provides an extremely large surface-area to volume ratio interfacing the two phases. Thus, the resulting fluid develops a notably higher thermal conductivity than the carrier liquid [2-11]. This enhances their performance in thermal management where the nanofluids form the cooling fluid, i.e., they remove heat from a cold-plate mated to a heat source and subsequently reject it to the ambient through a radiator [12, 13]. Extensive experimental studies have explored the dependence of the thermal conductivity on various parameters, e.g., NP volume fraction and temperature. It is now established that, for low NP volume fractions, the thermal conductivity enhancement is moderate and can be predicted by effective medium theories [14]. However, a liquid cooling system used in thermal management requires rapid flow of the nanofluid through closed conduits. Thus, design of such systems requires thorough knowledge of the flow characteristics of nanofluids. A particularly significant consideration is the fluid viscosity as heat transfer by convection is strongly correlated to viscosity. Thus, an exhaustive map of the dependence of viscosity on various parameters, e.g., NP volume fraction and temperature, is vital. Unfortunately, this has received limited attention to date [15]. Particularly, the viscosity variation of nanofluids containing an organic carrier fluid [16] are relatively unexplored.

Viscosity of a nanofluid is typically higher than the base or carrier fluid. For instance, $\mathrm{Al}_{2} \mathrm{O}_{3}$-water and $\mathrm{TiO}_{2}$-water nanofluids show a threefold increase in viscosity relative to water at a NP volume fraction $\phi=10 \%$ [10]. Viscosity typically increases monotonically with both NP size and volume fraction, e.g., $\mathrm{TiO}_{2}$-water [17] and the $\mathrm{SiO}_{2}$-ethanol $[18,19]$ nanofluids. Increasing volume fraction may lead to aggregation of NPs into bigger clusters; however, contribution of clustering is inconclusive in most studies.

Viscosity also depends on temperature [20, 21], but the dependence is often specific to material composition. For instance, nanofluids with $\mathrm{CuO}, \mathrm{Al}_{2} \mathrm{O}_{3}$ and $\mathrm{SiO}_{2} \mathrm{NPs}$ in alcohol-water solutions show an exponential decay in viscosity with temperature between $-35^{\circ} \mathrm{C}$ and $50^{\circ} \mathrm{C}$ [21], quite similar to the carrier fluids. On the contrary, nanofluids containing carbon nanotubes as the 
dispersed phase show that viscosity is independent of temperature between $0^{\circ} \mathrm{C}$ and $50^{\circ} \mathrm{C}$ and increase significantly beyond $55^{\circ} \mathrm{C}[20]$.

The effective viscosity of nanofluids is dependent on various physical, chemical and geometrical properties: $\eta_{\text {eff }}=f\left(\phi, T, L_{c}, t_{B}, t_{r}, B, \varepsilon\right)$ [19]. Assuming low volume fraction of nanoparticles, negligible effect of fluid and particle thermal conductivities, absence of perikinetic aggregation due to Brownian motion and orthokinetic aggregation arising from shearing in the fluid, we employ a dimensional analysis to predict the fundamental quantities that exert significant contributions on $\eta_{\text {eff }}$. Using the Buckingham Pi theorem [22], we obtain the dimensionless groups to be

$$
\begin{gathered}
\pi_{1}=\eta_{r} \\
\pi_{2}=\phi \\
\pi_{3}=\frac{\eta_{b} \gamma d^{2}}{k_{B} T}\left[\left(\frac{\phi_{m}}{\phi}\right)^{1 / 3}-1\right]
\end{gathered}
$$

Thus, the relative viscosity of nanofluids,

$$
\eta_{r}=f\left(\phi, \frac{\eta_{b} \gamma d^{2}}{k_{B} T}\left[\left(\frac{\phi_{m}}{\phi}\right)^{1 / 3}-1\right]\right)
$$

shows that nanoparticle volume fraction $\phi$ and temperature $T$ are two key parameters controlling nanofluid viscosity for a specific combination of fluid and NP.

Most models that describe dependence of viscosity on the different parameters can be traced back to Einstein's prediction based on Newtonian fluids and are applicable only for low particle volume fractions [1]. Recently, new correlations were proposed that considered temperature dependence of nanofluid viscosity by incorporating the effect of Brownian motion of the NPs using the Brinkman formulations [23]. This enabled prediction of viscosity even up to moderate NP concentrations. Other factors, e.g., the shape of the particle [24], have also been investigated in more sophisticated studies. However, the literature is quite scarce on predictive models for viscosity of nanofluids, especially for those based on organic solvents. In this letter, 
driven by the observations from our dimensionless analyses shown above, we examine the temperature and particle volume fraction dependence of viscosity for magnetite-toluene nanofluids and provide a predictive model to support results of our experimental measurements.

\section{Methods}

Toluene is considered as the carrier liquid because of its wide applications in cleaning agents, adhesives, resins, paints and paint thinners. Dry magnetite $\left(\mathrm{Fe}_{3} \mathrm{O}_{4}\right)$ NPs with a proprietary hydrophobic coating (EMG 1400, Ferrotec Inc., USA) are dispersed in toluene by a vortex shaker followed by heating the solution in a water bath at $40^{\circ} \mathrm{C}$ to enhance the mixing and particle dispersion in the fluid medium. Each sample is inspected under an optical microscope at $400 \times$ magnification to ensure no observable micro clusters are present.

\section{Results and Discussion}

Dynamic light scattering (DLS, Precision Detectors ${ }^{\mathrm{TM}}$ PD2000DLS ${ }^{\mathrm{PLUS}}$ ), carried out at room temperature $(T=298 \mathrm{~K})$, yields the hydrodynamic diameter of the particulate phase for two representative samples with volume fraction $\phi=0.01 \%$ and $0.05 \%$, as presented in Figure $1 \mathrm{~b}$. Figure 1a shows that the correlation function decays exponentially with time, i.e., the particulate phase is fairly monodisperse [25]. They also show that particles aggregate to form bigger clusters with increasing $\phi$. The dispersion with $\phi=0.01 \%$ has minimal clustering with mean diameter $a \approx d$, as determined by an approximate measurement of $d \sim 10 \mathrm{~nm}$ from the TEM images presented in Figure 1c. The dispersion with $\phi=\mathbf{0 . 0 5 \%}$, however, shows significant aggregation, yielding $a \approx 10 d$. As the clusters are fairly monodisperse, the viscosity of this nanofluid can be explained purely by considering either the volume fraction or the size of the dispersed phase, i.e., independent consideration of both is not required.

Viscosity of the nanofluids is measured with a sine wave vibro viscometer (SV-10, A\&D Japan). The viscometer transmits a uniform frequency to its two sensor plates maintained at constant amplitude by an electromagnetic force [26]. Viscosity is computed by correlating the driving electric current with the magnitude of the viscous force between the sensor plates and the fluid. This has the advantage of minimizing the energetic interference to a sample liquid unlike, e.g., a rotating drum viscometer, wherein such interference occurs by a rotative force and 
barycentric shift. Thus, it can be used for continuously measuring a wide range of viscosities in order to observe a viscosity change caused by a temperature change. The measured viscosities are shown in Figure 2 as a function of (a) temperature and (b) particle volume fraction. We obtain a viscosity of $0.45-0.52 \mathrm{mPa}$.s for pure toluene that agrees very well with the literature [27], thus validating our measurement method.

We see from Figures 2 that the effective nanofluid viscosity $\eta_{\text {eff }}$ increases with increasing NP volume fraction $\phi$. With higher $\phi$, there is a monotonic increase in particle cluster size $a$. Nanoparticles start agglomerating with increase in concentration and the fluid molecules in the interfacial zones between NPs become increasingly immobile at higher volume fractions. We find from the DLS particle size distribution in Figure 1 that at higher concentrations the average particle sizes are larger, supporting our prediction. Agglomeration also implies that a larger stress is necessary to break this attraction among particles; therefore, a high relative viscosity would be observed in the nanofluids as shown in Figure 2b. Hence, the particle aggregation in the nanofluids along with the solid-liquid interfacial effects contributes towards augmenting the shear viscosity.

Temperature dependence of viscosity, shown in Figure 2a, suggests that at high temperatures the viscosity augmenting effect of NPs gets diminished. The viscosity drop across the investigated temperature range is larger for higher $\phi$ nanofluids than for the lower concentration ones. With lowering of the temperature, the intermolecular interactions between particles decrease, which increases the nanofluid viscosity [15]. At higher temperatures, the higher kinetic energies of the molecules facilitate enhanced fluid motion and lower the viscosity [15]. The kinematic viscosity is influenced by particle kinetic energies, inherently reflecting particle accelerations and interactions. Additionally, fluid-particle interactions influence the thermo-physical properties of solid/liquid nano-suspensions by forming a 'nanolayer' that act as a thermal bridge between a solid nanoparticle and the liquid medium in case of thermal conductivity enhancement [5]. Additional investigation is however required to understand the effects of such nanolayers on viscosity enhancement in nanofluids.

Based on the measurements, we propose an empirical correlation describing the dependence of magnetite-toluene nanofluid viscosity on temperature and particle volume fraction: 


$$
\eta_{r}=\left(1+308.7 \phi+0.9649 \phi^{3}\right)\left(1-20.55 \exp \left(-36.94 T_{r}+137.7 T_{r}^{-1}-108.6 T_{r}^{-2}\right)\right)(5)
$$

where $\eta_{r}$ is the ratio of effective nanofluid viscosity to the viscosity of the base fluid at $T=298$ $\mathrm{K}, \phi$ is the particle volume fraction and $T_{r}=T / 298 \mathrm{~K}$ is the non-dimensional nanofluid temperature. This correlation is obtained by fitting the experimental data to a model inspired by the various popular forms [1], yielding $R^{2}=0.9224$ (coefficient of determination, pronounced $\mathrm{r}$ square). When higher order terms in $\phi$ are neglected, and at any given temperature, the correlation reduces to a form similar to the Einstein equation for low volume fractions [28]. However, our model, unlike the Einstein equation, does capture the dependencies on temperature and particle aggregation observed for higher volume fractions of the NPs, especially in an organic solvent.

\section{Conclusions}

In summary, we investigate the shear viscosity variation of magnetite-toluene nanofluids as a function of particle volume fraction and temperature. Our experimental measurements show that the nanofluid viscosity increases with the addition of nanoparticles, while it decreases with increasing temperature. Particle size distributions show the effect of aggregation at higher particle concentrations that contributes to stronger intermolecular forces retarding fluid motion. In the nanofluids investigated here, there is no means to vary particle concentration without simultaneously influencing particle aggregation. Thus, the influence of concentration and aggregation cannot be decoupled herein; it will be addressed in a future exercise using model nanofluids system providing independent control of both parameters. Increase in kinetic energies at higher temperatures enhances transport of fluid molecules lowering the viscosity. The rate of decrease with increasing temperature is lower at higher particle concentration. This suggests that temperature effects on particle suspension properties may be very different for high particle fraction than for lower ones. An empirical model is proposed for predicting the nanofluid viscosity incorporating the contributions of particle volume fraction and temperature. 


\section{List of Symbols}

$\begin{array}{ll}a & \text { particle cluster diameter } \\ B & \text { buoyancy force } \\ d & \text { nanoparticle diameter } \\ k_{B} & \text { Boltzmann constant, } 1.381 \times 10^{-23} \mathrm{~J} / \mathrm{K} \\ l & \text { distance between two neighboring particles } \\ L_{C} & \text { characteristic length } \\ t_{B} & \text { Brownian characteristic time } \\ t_{r} & \text { shearing characteristic time } \\ T & \text { temperature } \\ T_{r} & \text { dimensionless temperature }\end{array}$

Greek Letter

$\quad \quad \quad$ base/carrier fluid viscosity

$\eta_{\text {eff }} \quad$ effective viscosity of nanofluid

$\eta_{r} \quad$ relative viscosity of a nanofluid defined by $\eta_{r}=\eta_{\text {eff }} / \eta_{b}$ (dimensionless)

$\phi \quad$ nanoparticle volume fraction

$\phi_{m} \quad$ crowding factor

$\varepsilon \quad$ depth of van der Waals interaction potential

$\gamma \quad$ shear rate 


\section{Acknowledgments}

GB and OS thank the Iowa State University MOSAIC REU program supported through the NSF award EEC-1263243 for partial funding for this project. GB also acknowledges support of Center for Excellence in Learning and Teaching for the Miller Faculty Fellowship. RS would like to thank the College of Engineering for the Dean's Fellowship. 


\section{References}

[1] I.M. Mahbubul, R. Saidur, M.A. Amalina, International Journal of Heat and Mass Transfer, 55 (2012) 874-885.

[2] M.P. Beck, Y. Yuan, P. Warrier, A.S. Teja, Journal of Nanoparticle Research, 11 (2008) 1129-1136.

[3] R. Taylor, S. Coulombe, T. Otanicar, P. Phelan, A. Gunawan, W. Lv, G. Rosengarten, R. Prasher, H. Tyagi, Journal of Applied Physics, 113 (2013) 011301.

[4] M. Chandrasekar, S. Suresh, A. Chandra Bose, Experimental Thermal and Fluid Science, 34 (2010) 210-216.

[5] S.U.S. Choi, Z.G. Zhang, W. Yu, F.E. Lockwood, E.A. Grulke, Applied Physics Letters, 79 (2001) 2252.

[6] S.K. Das, N. Putra, P. Thiesen, W. Roetzel, Journal of Heat Transfer, 125 (2003) 567.

[7] T.-K. Hong, H.-S. Yang, C.J. Choi, Journal of Applied Physics, 97 (2005) 064311.

[8] J.-Y. Jung, C. Cho, W.H. Lee, Y.T. Kang, International Journal of Heat and Mass Transfer, 54 (2011) 1728-1733.

[9] S.M.S. Murshed, K.C. Leong, C. Yang, International Journal of Thermal Sciences, 44 (2005) 367-373.

[10] S.M.S. Murshed, K.C. Leong, C. Yang, International Journal of Thermal Sciences, 47 (2008) 560-568.

[11] H. Xie, J. Wang, T. Xi, Y. Liu, F. Ai, Q. Wu, Journal of Applied Physics, 91 (2002) 4568.

[12] Y. He, Y. Jin, H. Chen, Y. Ding, D. Cang, H. Lu, International Journal of Heat and Mass Transfer, 50 (2007) 2272-2281.

[13] L.S. Sundar, K.V. Sharma, International Journal of Heat and Mass Transfer, 53 (2010) 4280-4286.

[14] J. Buongiorno, D.C. Venerus, N. Prabhat, T. McKrell, J. Townsend, R. Christianson, Y.V. Tolmachev, P. Keblinski, L.-w. Hu, J.L. Alvarado, I.C. Bang, S.W. Bishnoi, M. Bonetti, F. Botz, A. Cecere, Y. Chang, G. Chen, H. Chen, S.J. Chung, M.K. Chyu, S.K. Das, R. Di Paola, Y. Ding, F. Dubois, G. Dzido, J. Eapen, W. Escher, D. Funfschilling, Q. Galand, J. Gao, P.E. Gharagozloo, K.E. Goodson, J.G. Gutierrez, H. Hong, M. Horton, K.S. Hwang, C.S. Iorio, S.P. Jang, A.B. Jarzebski, Y. Jiang, L. Jin, S. Kabelac, A. Kamath, M.A. Kedzierski, L.G. Kieng, C. Kim, J.-H. Kim, S. Kim, S.H. Lee, K.C. Leong, I. Manna, B. Michel, R. Ni, H.E. Patel, J. Philip, 
D. Poulikakos, C. Reynaud, R. Savino, P.K. Singh, P. Song, T. Sundararajan, E. Timofeeva, T. Tritcak, A.N. Turanov, S. Van Vaerenbergh, D. Wen, S. Witharana, C. Yang, W.-H. Yeh, X.-Z. Zhao, S.-Q. Zhou, Jouurnal of Applied Physics, 106 (2009) 094312.

[15] C. Nguyen, F. Desgranges, G. Roy, N. Galanis, T. Mare, S. Boucher, H. Angue Mintsa, International Journal of Heat and Fluid Flow, 28 (2007) 1492-1506.

[16] D. Li, W. Xie, W. Fang, Nanoscale Research Letters, 6 (2011) 373.

[17] A. Turgut, I. Tavman, M. Chirtoc, H.P. Schuchmann, C. Sauter, S. Tavman, International Journal of Thermophysics, 30 (2009) 1213-1226.

[18] J. Chevalier, O. Tillement, F. Ayela, Applied Physics Letters, 91 (2007) 233103.

[19] J. Chevalier, O. Tillement, F. Ayela, Physical Review E, 80 (2009).

[20] L. Chen, H. Xie, Y. Li, W. Yu, Thermochimica Acta, 477 (2008) 21-24.

[21] D.P. Kulkarni, D.K. Das, R.S. Vajjha, Applied Energy, 86 (2009) 2566-2573.

[22] E. Buckingham, Physical Review, 4 (1914) 345-376.

[23] Y. Bakhshan, M. Saljooghi, Journal of Computational Theoritical Nanoscience, 11 (2014) 583-588.

[24] G. Balasubramanian, S. Sen, I.K. Puri, Physics Letters A, 376 (2012) 860-863.

[25] W. Brown, Dynamic light scattering: the method and some applications, Oxford University Press, USA, 1993.

[26] N. Izumo, A. Koiwai, in: Proceedings of Asia-Pacific Symposium on Measurement of Mass, Force and Torque, Tokyo, Japan, 2009, pp. 51-57.

[27] F.J.V. Santos, Journal of Physical and Chemical Reference Data, 35 (2006) 1.

[28] A. Einstein, Annalen der Physik, 324 (1906) 289-306. 

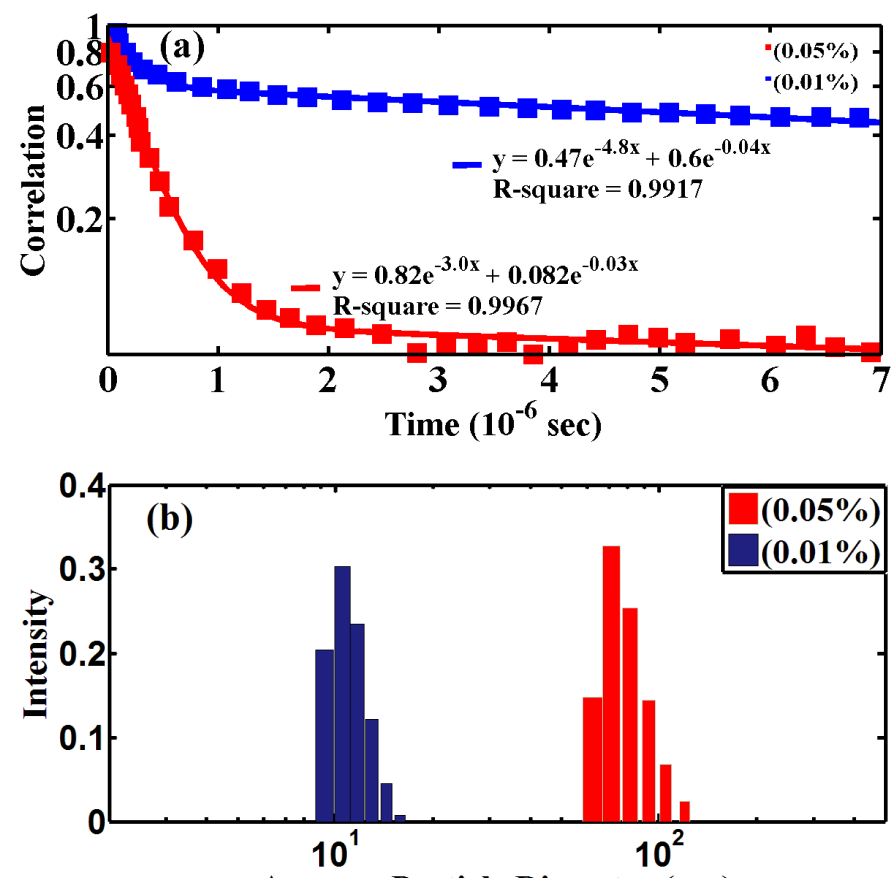

Average Particle Diameter (nm)

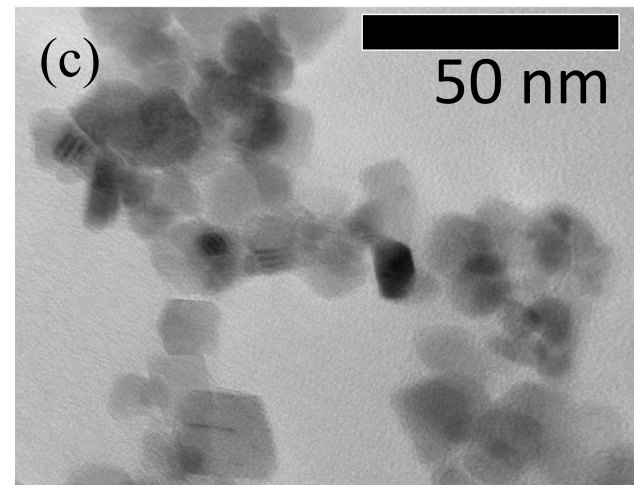

Figure 1. Comparison of dynamic light scattering results for two samples, Sample 1 (0.01\%) and Sample 5 (0.05\%): (a) The correlation function shows an exponential decay for both samples, establishing that the particle sizes are fairly monodisperse [25]. (b) Particle size distribution for both samples, determined from DLS, shows that as the concentration of nanoparticles is increased, the particles agglomerate to increase the hydrodynamic diameter from $10 \mathrm{~nm}$ (Sample 1) to around $100 \mathrm{~nm}$ (Sample 5). (c) TEM micrograph of the nanoparticles show that individual particles have a diameter $\sim 10 \mathrm{~nm}$. 

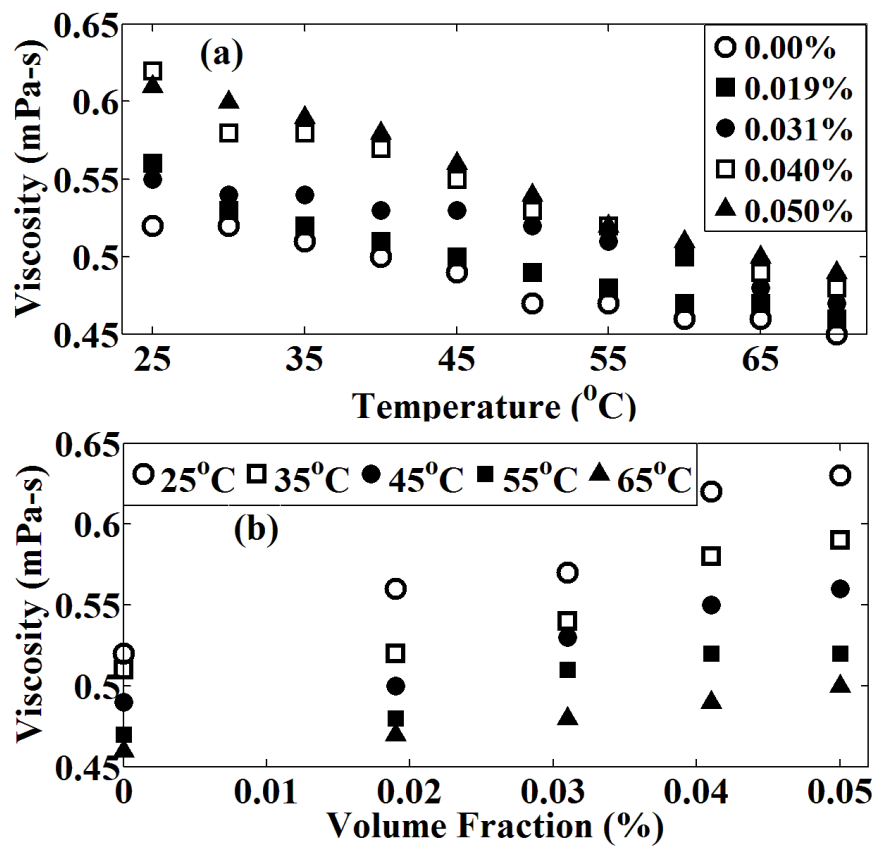

Figure 2. Nanofluid viscosity presented as a function of temperature and volume fraction.

(a) Results are shown for five different particle volume fractions varying from $0 \%$ to 0.05\%. Viscosity increases with the increase in particle concentrations and it remains higher than the base fluid at all the temperatures in the range of $25^{\circ} \mathrm{C}-70^{\circ} \mathrm{C}$. With increasing temperature viscosity decreases linearly for low concentrations. Figure (b) shows the increase of viscosity with volume fraction at different temperatures. 